\title{
Características eletrofisiológicas das pessoas diabéticas com síndrome do túnel do carpo*
}

\section{Electrodiagnostic Testing Characteristics of Diabetic People with Carpal Tunnel Syndrome}

\author{
Henver Ribeiro Paiva Filho ${ }^{10}$ Alex Timóteo Rodrigues Reis ${ }^{2}$ Gabriel Antonio Matos ${ }^{2}$ \\ Valdênia Graças Nascimento Paiva ${ }^{3(-)}$ Elias Felix Oliveira ${ }^{4(-)}$ Murilo Antônio Rocha ${ }^{5(0)}$
}

1 Universidade Federal do Triângulo Mineiro, Uberaba, MG, Brasil

2 Serviço de Ortopedia e Traumatologia, Universidade Federal do

Triângulo Mineiro, Uberaba, MG, Brasil

${ }^{3}$ Cirurgia da Mão, Serviço de Ortopedia e Traumatologia,

Universidade Federal do Triângulo Mineiro, Uberaba, MG, Brasil

${ }^{4}$ Serviço de Ortopedia e Traumatologia, Universidade Federal do

Triângulo Mineiro, Uberaba, MG, Brasil

${ }^{5}$ Cirurgia do Pé, Serviço de Ortopedia e Traumatologia, Universidade

Federal do Triângulo Mineiro, Uberaba, MG, Brasil

\begin{abstract}
Endereço para correspondência Valdênia das Graças Nascimento Paiva, MD, Ambulatório Maria da Glória - Ortopedia e Traumatologia, Hospital de Clínicas da Universidade Federal do Triângulo Mineiro, Uberaba, MG, Brasil (e-mail: vallfmtm@yahoo.com.br).
\end{abstract}

Rev Bras Ortop 2021;56(3):356-359.

\section{Resumo \\ Palavras-chave \\ - diabetes \\ - eletromiografia \\ - neuropatia mediana \\ - parestesia \\ - síndrome do túnel do carpo}

Objetivo O presente trabalho teve por objetivo verificar se existe correlação entre a síndrome do túnel do carpo (STC) e eletroneuromiografia (ENMG) de pacientes diabéticos e não diabéticos.

Métodos Foram avaliados 154 pacientes em um ambulatório de cirurgia da mão. Todas as ENMGs avaliadas foram realizadas por um único neurologista, bilateralmente. As variáveis qualitativas foram descritas para todas as pessoas em acompanhamento devido à STC segundo a presença de diabetes e foi verificada a associação com uso do teste qui-quadrado. Foi ajustado o modelo conjunto para verificar a influência da diabetes na gravidade da ENMG em pacientes com STC.

Resultados Foram incluídos no presente estudo 117 mulheres e 37 homens, com média de idade de 56,9 anos. Eletroneuromiografia demonstrando STC bilateral foi observada em $82,5 \%$ das pessoas. Pessoas diabéticas foram identificadas em $21,4 \%$ dos casos. Eletroneuromiografia com padrão grave foi prevalente.

Conclusão Não houve associação entre a presença de diabetes e a gravidade da ENMG em pessoas com STC. Nível de evidência IV, série de casos.

\footnotetext{
Trabalho feito no Serviço de Ortopedia e Traumatologia, Cirurgia da Mão, Universidade Federal do Triângulo Mineiro, Uberaba, MG, Brasil.
}

recebido

06 de Maio de 2020

aceito

16 de Setembro de 2020

Publicado on-line

Fevereiro 10, 2021
DOI https://doi.org/ 10.1055/s-0040-1721841. ISSN 0102-3616.

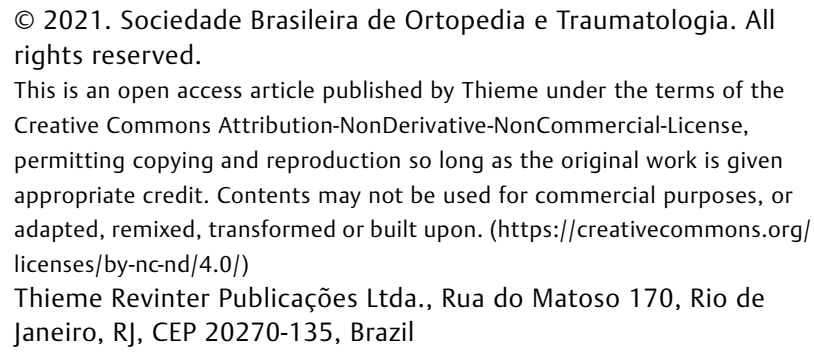

(c) 2021. Sociedade Brasileira de Ortopedia e Traumatologia. All rights reserved.

This is an open access article published by Thieme under the terms of the Creative Commons Attribution-NonDerivative-NonCommercial-License, permitting copying and reproduction so long as the original work is given appropriate credit. Contents may not be used for commercial purposes, or adapted, remixed, transformed or built upon. (https://creativecommons.org/ licenses/by-nc-nd/4.0/) Thieme Revinter Publicações Ltda., Rua do Matoso 170, Rio de Janeiro, RJ, CEP 20270-135, Brazil 


Abstract
Keywords
- diabetes
- electromyography
- median
neuropathy
- paresthesia
- carpal tunnel
syndrome

Objective The present study aimed to correlate electroneuromyography (ENMG) findings in diabetic and nondiabetic subjects with carpal tunnel syndrome (CTS).

Methods In total, 154 patients were evaluated in a hand surgery outpatient clinic. All ENMG tests were bilaterally performed by a single neurologist. Qualitative variables were described for all patients with CTS according to their diabetic status, and the chisquared test was used to reveal any association. A joint model was adjusted to determine the influence of diabetes on ENMG severity in CTS patients.

Results The sample consisted of 117 women and 37 men, with an average age of 56.9 years old. Electroneuromyography demonstrated bilateral CTS in $82.5 \%$ of the patients. Diabetes was identified in $21.4 \%$ of the cases. Severe ENMG was prevalent.

Conclusion There was no association between diabetes and ENMG severity in patients with CTS. Level of evidence IV, case series.

\section{Introdução}

A síndrome do túnel do carpo (STC) é uma neuropatia comum no paciente diabético, com acometimento estimado em $14 \%$ nos diabéticos sem neuropatia e em $30 \%$ naqueles com neuropatia diabética. ${ }^{1,2}$ A STC é frequente no paciente diabético não somente pela alteração do tecido sinovial circundante, mas também por o nervo apresentar alterações secundárias à glicemia elevada. ${ }^{3}$

As complicações neurológicas mais comuns em diabéticos são as polineuropatias simétricas sensorimotoras e as neuropatias focais, sendo as neuropatias compressivas da extremidade superior as mais frequentes em qualquer estágio da diabetes. ${ }^{4}$

A possível patogênese da STC na população diabética envolve o aumento de citocinas inflamatórias circulantes decorrentes do produto final do processo de glicação, resultando em desmielinização e perda axonal dos nervos periféricos, o que torna os nervos, dentre eles o mediano, mais suscetíveis à compressão. ${ }^{5}$

Encontramos na literatura estudos demonstrando características das pessoas diabéticas, com e sem STC; ${ }^{3}$ entretanto, ainda são escassas publicações recentes que demonstrem as características clínicas, epidemiológicas e eletroneuromiográficas de pessoas em acompanhamento ambulatorial devido a STC, com e sem diabetes. O objetivo do presente trabalho foi verificar se existe diferença das características clínicas e dos resultados da eletroneuromiografia (ENMG) em pessoas com STC, diabéticas e não diabéticas.

\section{Casuística e Métodos}

Estudo transversal realizado em um ambulatório de cirurgia da mão com avaliação de 154 pacientes. Realizamos os procedimentos de acordo com o Comitê de Ética em Pesquisa sob o número de autorização 3.640.789, e com a Declaração de Helsinque de 1964. Obtivemos o Termo de Consentimento Livre e Esclarecido de todos os participantes.

Incluímos pessoas de ambos os gêneros, $>18$ anos de idade, que apresentavam ENMG de membros superiores positiva para STC. Excluímos gestantes, pessoas com diabetes tipo I ou com cirurgias prévias no punho.

Todas as ENMGs avaliadas foram realizadas por um único neurologista, sem vínculo com este trabalho, bilateralmente, e foi utilizado o eletroneuromiógrafo modelo Neuropack EMG (S1, MEB-9400K, Nihon Kohden Corporation, Tóquio, Japão).

A ENMG foi classificada de acordo com a classificação de Stevens em leve (alteração somente da condução sensitiva), moderada (alteração da condução sensitiva e motora) e grave (condução sensitiva e motora alteradas e sinais de denervação à eletromiografia de agulha). ${ }^{6}$

A variável quantitativa idade foi descrita por meio de média e desvio padrão (DP). As qualitativas foram descritas para todas as pessoas em acompanhamento devido a STC que apresentavam ENMG positiva e segundo a presença de diabetes, e foi verificada a associação com uso do teste quiquadrado. $^{7}$

Foram atendidas 159 pessoas com ENMG positiva para STC, e 5 foram excluídas devido à descrição incompleta do laudo, totalizando 154 participantes incluídos no presente estudo.

Em relação ao gênero, foram incluídas 117 (76,0\%) mulheres e 37 homens (24,0\%). A média e o DP da idade foi 56,9 $\pm 10,9$ anos. Índice de massa corporal (IMC) dentro do padrão da normalidade foi encontrado em $71(46,1 \%)$ pessoas, sobrepeso em 51 (33,1\%) e obesidade em 32 (20,8\%). Eletroneuromiografia demonstrando STC bilateral foi observada em 127 (82,5\%) pessoas, e unilateral em 27 (17,5\%). Um total de 33 $(21,4 \%)$ pessoas apresentavam o diagnóstico de diabetes e 121 (78,6\%) não. A presença de somente 1 doença sistêmica além da diabetes, e a presença de $\geq 2$ doenças, foi igualmente encontrada em 52 (33,8\% cada) pessoas, enquanto 50 $(32,4 \%)$ não apresentavam comorbidades.

Para a análise estatística, foi ajustado o modelo conjunto para verificar a influência da diabetes na gravidade da ENMG em pacientes com STC, sendo inseridas no modelo as variáveis que apresentaram nível descritivo $<0,20$ nos testes bivariados $(p<0,20)$. Os testes foram realizados com nível de significância de $5 \%$. 
Tabela 1 Características das pessoas com síndrome do túnel do carpo e diabetes

\begin{tabular}{|c|c|c|c|}
\hline \multirow[t]{2}{*}{ Variável } & \multicolumn{2}{|l|}{ Diabetes } & \multirow[t]{2}{*}{ valor-p } \\
\hline & $\begin{array}{l}\text { Não } \\
(n=121)\end{array}$ & $\begin{array}{l}\operatorname{Sim} \\
(n=33)\end{array}$ & \\
\hline Idade (anos) & & & $0,244^{*}$ \\
\hline média \pm DP & $56,4 \pm 10,9$ & $58,9 \pm 11$ & \\
\hline $\begin{array}{l}\text { mediana } \\
\text { (mín.; máx.) }\end{array}$ & $56(30 ; 84)$ & $58(33 ; 89)$ & \\
\hline Gênero,n (\%) & & & 0,023 \\
\hline Feminino & $87(71,9)$ & $30(90,9)$ & \\
\hline Masculino & $34(28,1)$ & $3(9,1)$ & \\
\hline $\begin{array}{l}\text { IMC } \\
\text { classificação,n (\%) }\end{array}$ & & & 0,121 \\
\hline Normal & $61(50,4)$ & $10(30,3)$ & \\
\hline Sobrepeso & $37(30,6)$ & $14(42,4)$ & \\
\hline Obesidade & $23(19)$ & $9(27,3)$ & \\
\hline $\begin{array}{l}\text { ENMG } \\
\text { lateralidade,n (\%) }\end{array}$ & & & 0,150 \\
\hline Unilateral & $24(19,8)$ & $3(9,1)$ & \\
\hline Bilateral & $97(80,2)$ & $30(90,9)$ & \\
\hline $\begin{array}{l}\text { Doenças } \\
\text { sistêmicas,n (\%) }\end{array}$ & & & $<0,001$ \\
\hline 1 & $50(41,3)$ & $2(6,1)$ & \\
\hline$\geq 2$ & $21(17,4)$ & $31(93,9)$ & \\
\hline Não & $50(41,3)$ & $0(0)$ & \\
\hline
\end{tabular}

Abreviações: DP, desvio padrão; ENMG, eletroneuromiografia; IMC, índice de massa corporal.

Teste qui-quadrado.

*Teste $\mathrm{t}$ de Student.

\section{Resultados}

Quanto à classificação da ENMG, o padrão leve foi observado em 18 (11,7\%) pessoas, enquanto o moderado foi observado em 64 (41,6\%), e o grave em 72 (46,7\%).

A - Tabela 1 mostra que, de todas as mulheres com STC, a frequência nas com diabetes foi maior do que naquelas sem diabetes $(p=0,023)$. Houve também maior frequência de doenças sistêmicas associadas nos pacientes diabéticos do que naqueles não diabéticos $(p<0,001)$.

A - Tabela 2 mostra que apenas a lateralidade da STC apresentou associação com o grau da STC ( $p<0,001)$, sendo que pacientes com STC bilateral apresentaram graus mais graves da ENMG.

A presença de diabetes não apresentou associação estatística com o grau da ENMG $(p=0,466)$.

Pela - Tabela 3, observa-se que a lateralidade da STC à ENMG sofreu influência com significância estatística $(p=0,023)$. Pacientes com STC bilateral apresentaram graus de ENMG 44\% mais graves do que pacientes com exame positivo unilateralmente.

A presença de diabetes não influenciou o grau da ENMG para a STC $(p=0,927)$.
Tabela 2 Descrição dos graus da síndrome do túnel do carpo segundo as características avaliadas e resultado dos testes estatísticos

\begin{tabular}{|c|c|c|c|c|}
\hline \multirow[t]{2}{*}{ Variável } & \multicolumn{3}{|c|}{ ENMG classificação } & \multirow[t]{2}{*}{ valor $-p$} \\
\hline & Leve & Moderada & Grave & \\
\hline Idade (anos) & & & & $0,081^{*}$ \\
\hline média \pm DP & $56,3 \pm 10,6$ & $54,8 \pm 10,8$ & $58,9 \pm 10,8$ & \\
\hline $\begin{array}{l}\text { mediana } \\
\text { (mín.; máx.) }\end{array}$ & $\begin{array}{l}54,5 \\
(42 ; 83)\end{array}$ & $\begin{array}{l}55 \\
(30 ; 81)\end{array}$ & $\begin{array}{l}58 \\
(41 ; 89)\end{array}$ & \\
\hline Gênero,n (\%) & & & & 0,373 \\
\hline Feminino & $14(12)$ & $45(38,5)$ & $58(49,6)$ & \\
\hline Masculino & $4(10,8)$ & $19(51,4)$ & $14(37,8)$ & \\
\hline $\begin{array}{l}\text { IMC } \\
\text { classificação, } \\
n(\%)\end{array}$ & & & & 0,821 \\
\hline Normal & $8(11,3)$ & $31(43,7)$ & $32(45,1)$ & \\
\hline Sobrepeso & $6(11,8)$ & $18(35,3)$ & $27(52,9)$ & \\
\hline Obesidade & $4(12,5)$ & $15(46,9)$ & $13(40,6)$ & \\
\hline $\begin{array}{l}\text { ENMG } \\
\text { lateralidade, } \\
n(\%)\end{array}$ & & & & $<0,001$ \\
\hline Unilateral & $10(37)$ & $15(55,6)$ & $2(7,4)$ & \\
\hline Bilateral & $8(6,3)$ & $49(38,6)$ & $70(55,1)$ & \\
\hline $\begin{array}{l}\text { Doenças } \\
\text { sistêmicas, } \\
\mathrm{n}(\%)\end{array}$ & & & & 0,516 \\
\hline 1 & $7(13,5)$ & $19(36,5)$ & $26(50)$ & \\
\hline$\geq 2$ & $4(7,7)$ & $21(40,4)$ & $27(51,9)$ & \\
\hline Não & $7(14)$ & $24(48)$ & $19(38)$ & \\
\hline Diabetes & & & & 0,466 \\
\hline Não & $16(13,2)$ & $50(41,3)$ & $55(45,5)$ & \\
\hline $\operatorname{Sim}$ & $2(6,1)$ & $14(42,4)$ & $17(51,5)$ & \\
\hline
\end{tabular}

Abreviações: DP, desvio padrão; ENMG, eletroneuromiografia; IMC, índice de massa corporal.

Teste qui-quadrado.

*ANOVA.

Tabela 3 Resultado do modelo para explicar o grau da eletroneuromiografia para síndrome do túnel do carpo segundo as características avaliadas

\begin{tabular}{|l|l|l|l|l|}
\hline Variável & Coeficiente & \multicolumn{2}{|l|}{ IC (95\%) } & \multirow{2}{*}{ valor-p } \\
\cline { 3 - 4 } & & Inferior & Superior & \\
\hline $\begin{array}{l}\text { ENMG } \\
\text { (bilateral) }\end{array}$ & 1,44 & 1,05 & 1,98 & $\mathbf{0 , 0 2 3}$ \\
\hline Diabetes & 1,01 & 0,79 & 1,30 & 0,927 \\
\hline Idade (anos) & 1,001 & 0,991 & 1,011 & 0,836 \\
\hline
\end{tabular}

Abreviações: ENMG, eletroneuromiografia; IC, intervalo de confiança. Modelo linear generalizado (MLG) com distribuição Poisson e função de ligação logarítmica.

\section{Discussão}

Em estudos de base populacional, a prevalência da STC é maior em mulheres e aumenta com o avançar da idade, ${ }^{4}$ com estimativa de acometimento até três vezes maior no sexo masculino. ${ }^{8}$ 
De acordo com Papanas et al., ${ }^{9}$ a prevalência da STC em diabéticos varia entre 11 e $25 \%$, sendo mais comum também em mulheres. Nossos resultados foram condizentes com a literatura, com prevalência da STC em mulheres e média de idade de 56 anos em não diabéticos e de 58 anos em diabéticos.

Phalen ${ }^{10}$ refere que, nos diabéticos, o nervo mediano pode ser mais suscetível à compressão dentro do túnel do carpo quando comparado ao paciente sem diabetes. Na década de 1960, Mulder et al. ${ }^{11}$ encontraram prevalência de $9 \%$ da diabetes em pessoas com STC, e Blodgett et al. ${ }^{12}$ encontraram prevalência de 6,4\%. Em 1985, Comi et al. ${ }^{13}$ observaram 7,7\%, e Kouyoumdjian ${ }^{14}$ observou $4,4 \%$. A quantidade de diabéticos em nossos resultados (21,4\%) foi consideravelmente superior à literatura, o que acreditamos ser devido ao nosso serviço ser de referência regional, com número elevado de casos.

De acordo com Becker et al., ${ }^{15}$ o IMC elevado é fator de risco para STC, assim como sexo feminino, idade entre 40 e 60 anos e presença de diabetes. Para Bland, ${ }^{16}$ pessoas diabéticas com STC apresentam maior frequência de sobrepeso e obesidade do que aquelas com IMC dentro da normalidade. Assim como os autores acima, nosso estudo apresentou resultados semelhantes, visto que a prevalência foi de mulheres com idade de 56 anos. Apesar de 69,7\% dos diabéticos com STC apresentarem sobrepeso ou obesidade, não houve diferença no grupo dos não diabéticos (49,6\%).

Spahn et al. ${ }^{17}$ encontraram acometimento bilateral da STC em 50 a 60\% dos casos. Em nossos resultados, $82,46 \%$ dos pacientes apresentaram alteração eletroneuromiográfica bilateralmente, sendo que $44 \%$ destes apresentaram ENMG de grau grave; entretanto, não houve correlação entre a lateralidade e a gravidade da ENMG $(p=0,023)$.

Obtivemos um número considerável de pessoas que possuíam doenças sistêmicas comuns, dentre elas hipertensão, doenças reumatológicas e cardiológicas (67,6\%), e esse resultado teve significância entre diabéticos e não diabéticos. Em contrapartida, não foi encontrada diferença estatística nos resultados da ENMG entre pessoas diabéticas e não diabéticas com STC $(p=0,927)$. Isso pode estar relacionado à heterogeneidade do grupo avaliado, uma vez que idade, sexo feminino, IMC elevado, tempo de evolução e controle clínico adequado ou não se mostraram como fatores de risco independentes para STC.

A avaliação de pessoas com STC em um serviço de referência regional, para avaliar a relação entre diabetes e a gravidade da ENMG, é um dos pontos positivos do nosso estudo.

Uma das limitações do presente trabalho pode estar relacionada ao fato de que os pacientes com STC podem não representar a real influência da diabetes na gravidade da ENMG para avaliação desta afecção, visto que muitos diabéticos permanecem em acompanhamento na especialidade endocrinologia.

Também não é possível afirmar, peremptoriamente, que haja relação entre a maior gravidade dos achados eletroneuromiográficos e a bilateralidade da STC, sendo necessários novos trabalhos com espaço amostral maior e critérios de inclusão mais abrangentes para avaliar esta relação.

\section{Conclusão}

Concluímos que a STC é prevalente em mulheres na 5ª década de vida, tanto em diabéticas quanto não diabéticas, e que não existe associação entre a presença de diabetes e a gravidade da ENMG em pessoas com STC.

\section{Suporte Financeiro}

Não houve suporte financeiro de fontes públicas, comerciais, ou sem fins lucrativos.

Conflito de Interesses

Os autores declaram não haver conflito de interesses.

\section{Referências}

1 Ibrahim I, Khan WS, Goddard N, Smitham P. Carpal tunnel syndrome: a review of the recent literature. Open Orthop J 2012;6:69-76

2 Perkins BA, Olaleye D, Bril V. Carpal tunnel syndrome in patients with diabetic polyneuropathy. Diabetes Care 2002;25(03):565-569

3 Marciano LHC, Leite VM, Araujo PM, Garbino JA. Avaliação do comprometimento neurológico e da prevalência da síndrome do túnel do carpo em pacientes portadores de diabetes mellitus tipo 2. Acta Fisiatr 2007;14(03):134-141

4 Mondelli M, Aretini A, Rossi S. Ulnar neuropathy at the elbow in diabetes. Am J Phys Med Rehabil 2009;88(04):278-285

5 Mojaddidi MA, Ahmed MS, Ali R, et al. Molecular and pathological studies in the posterior interosseous nerve of diabetic and nondiabetic patients with carpal tunnel syndrome. Diabetologia 2014;57(08):1711-1719

6 Stevens JCAmerican Association of Electrodiagnostic Medicine. AAEM minimonograph \#26: the electrodiagnosis of carpal tunnel syndrome. Muscle Nerve 1997;20(12):1477-1486

7 Kirkwood BR, Sterne JA. Essential medical statistics. 2nd ed. Blackwell ScienceMassachusetts2006

8 Sassi SA, Giddins G. Gender differences in carpal tunnel relative cross-sectional area: a possible causative factor in idiopathic carpal tunnel syndrome. J Hand Surg Eur Vol 2016;41(06):638-642

9 Papanas N, Maltezos E. The diabetic hand: a forgotten complication? J Diabetes Complications 2010;24(03):154-162

10 Phalen GS. The carpal-tunnel syndrome. Clinical evaluation of 598 hands. Clin Orthop Relat Res 1972;83(83):29-40

11 Mulder DW, Lambert EH, Bastron JA, Sprague RG. The neuropathies associated with diabetes mellitus. A clinical and electromyographic study of 103 unselected diabetic patients. Neurology 1961;11(04):275-284

12 Blodgett RC Jr, Lipscomb PR, Hill RW. Incidence of hematologic disease in patients with carpal tunnel syndrome. JAMA 1962; 182:814-815

13 Comi G, Lozza L, Galardi G, Ghilardi MF, Medaglini S, Canal N. Presence of carpal tunnel syndrome in diabetics: effect of age, sex, diabetes duration and polyneuropathy. Acta Diabetol Lat 1985;22 (03):259-262

14 Kouyoumdjian JA. Síndrome do túnel do carpo: aspectos clínicoepidemiológico em 668 casos. Arq Neuropsiquiatr 1999;57(02): 504-512

15 Becker J, Nora DB, Gomes I, et al. An evaluation of gender, obesity, age and diabetes mellitus as risk factors for carpal tunnel syndrome. Clin Neurophysiol 2002;113(09):1429-1434

16 Bland JD. The relationship of obesity, age, and carpal tunnel syndrome: more complex than was thought? Muscle Nerve 2005;32(04):527-532

17 Spahn G, Wollny J, Hartmann B, Schiele R, Hofmann GO. [Metaanalysis for the evaluation of risk factors for carpal tunnel syndrome (CTS) Part I. General factors]. Z Orthop Unfall 2012;150(05):503-515 